\title{
Beyond Standard Model searches in B decays with ATLAS
}

\author{
Semen Turchikhin*, on behalf of the ATLAS collaboration \\ Joint Institute for Nuclear Research, Dubna, Russia \\ E-mail: Semen.Turchikhinecern.ch
}

\begin{abstract}
This contribution presents recent results of the ATLAS experiment at the LHC on heavy flavour measurements sensitive to possible contributions of the new physics. Two measurements are overviewed: the angular analysis of $B^{0} \rightarrow \mu^{+} \mu^{-} K^{* 0}$ decay and the measurement of the relative width difference between the eigenstates of the $B^{0}-\bar{B}^{0}$ system. The first one uses a data sample with an integrated luminosity of $20.3 \mathrm{fb}^{-1}$ collected by ATLAS at a centre of mass energy $\sqrt{s}=$ $8 \mathrm{TeV}$, and the second one benefits from the full ATLAS Run-1 dataset with additional $4.9 \mathrm{fb}^{-1}$ collected at $\sqrt{s}=7 \mathrm{TeV}$.
\end{abstract}

The International Conference on B-Physics at Frontier Machines - BEAUTY2018

6-11 May, 2018

La Biodola, Elba Island, Italy

\footnotetext{
* Speaker.
} 


\section{Introduction}

Studying heavy flavour decay processes at collider experiments is known to provide a means for indirect new physics (NP) searches. A number of such processes are forbidden at the lowest perturbative order and involve electroweak loop diagrams. Their properties are thus sensitive to possible NP contributions in the loops which may be comparable to the effects predicted by the Standard Model (SM). Precise measurement of these properties provides a test for the SM calculations and allows to constrain the parameter space of the corresponding NP models.

This paper overviews two recent measurements performed by the ATLAS experiment [1] at the Large Hadron Collider. The first one is the measurement of angular parameters of $B^{0} \rightarrow \mu^{+} \mu^{-} K^{* 0}$ decay [2]. Earlier the LHCb experiment reported 3.4 $\sigma$ deviation from the SM expectation in this decay [3], hence motivating further studies.

The other analysis performs the measurement of decay width relative difference in the $B^{0}-\bar{B}^{0}$ system, $\Delta \Gamma_{d} / \Gamma_{d}$ [4]. The SM predicts the value of $\Delta \Gamma_{d} / \Gamma_{d}=(0.42 \pm 0.08) \times 10^{-2}$ [5] while the uncertainty of the world average $\left(\Delta \Gamma_{d} / \Gamma_{d}=(0.1 \pm 1.0) \times 10^{-2}\right.$ as of 2014 [6] $)$ was too large to perform a stringent test of the prediction. It was also shown that independent measurements of other quantities do not constrain the value of $\Delta \Gamma_{d}$ and its relatively large variation due to possible NP contribution would not contradict other existing SM tests. Therefore the measurement of $\Delta \Gamma_{d}$ with improved precision can provide an independent test complementary to other NP searches.

\section{Angular analysis of $B^{0} \rightarrow \mu^{+} \mu^{-} K^{* 0}$ decay}

The analysis of the $B^{0} \rightarrow \mu^{+} \mu^{-} K^{* 0}$ decay is performed using the $p p$ collisions dataset collected by the ATLAS experiment at a centre of mass energy $\sqrt{s}=8 \mathrm{TeV}$ corresponding to an integrated luminosity of $20.3 \mathrm{fb}^{-1}$. Various trigger selection options based on search for single, di-, and tri-muon signatures are used in order to maximise the data statistics available for the analysis.

The kinematics of the $B^{0} \rightarrow \mu^{+} \mu^{-} K^{* 0}$ decay $^{1}$ (with subsequent $J / \psi \rightarrow \mu^{+} \mu^{-}$and $K^{* 0} \rightarrow$ $K^{+} \pi^{-}$) is described in terms of four variables: the dimuon invariant mass squared, $q^{2}$, and three angles describing the final state geometrical configuration, as illustrated in Fig. 1.

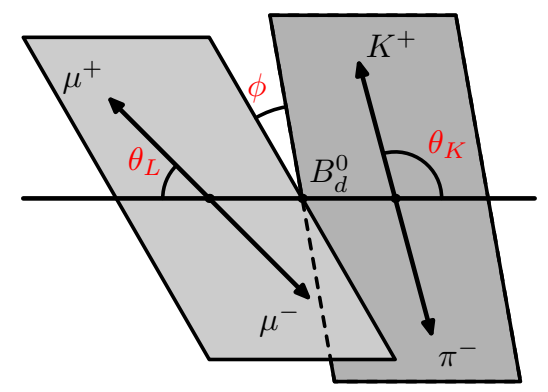

Figure 1: Illustration of the kinematic angles $\left(\phi, \theta_{L}, \theta_{K}\right)$ definition in $B^{0} \rightarrow \mu^{+} \mu^{-} K^{* 0}\left(K^{+} \pi^{-}\right)$decay. The polar angle $\theta_{K}\left(\theta_{L}\right)$ is defined in the $K^{* 0}$ (muon pair) rest frame, $\phi$ is the angle between the $K^{* 0}$ decay plane and the dimuon plane.

\footnotetext{
${ }^{1}$ Charge conjugation is implied here and below unless stated otherwise.
} 
The $B^{0}$ decay candidates are reconstructed by fitting a common vertex of two oppositely charged muon tracks and two other oppositely charged tracks forming a $K^{* 0}$ candidate. Signal candidate selection mostly aims at suppression of large combinatorial background. Selection criteria include a number of cuts on kinematic properties, quality of the vertex fit, and displacement of the decay vertex. Only the candidates with the $q^{2}$ value within the range of $[0.04,6] \mathrm{GeV}^{2}$ are retained for further signal fits; additionally, the range of $q^{2} \in[0.98,1.1] \mathrm{GeV}^{2}$ is excluded due to background from $\phi \rightarrow \mu^{+} \mu^{-}$decays. In the signal $q^{2}$ range, 787 signal candidates are found.

The differential decay rate as a function of the decay angles can be expressed as

$$
\begin{aligned}
\frac{1}{\mathrm{~d} \Gamma / \mathrm{d} q^{2}} \frac{\mathrm{d}^{4} \Gamma}{\mathrm{d} \cos \theta_{L} \mathrm{~d} \cos \theta_{K} \mathrm{~d} \phi \mathrm{d} q^{2}}= & \frac{9}{32 \pi}\left[\frac{3\left(1-F_{L}\right)}{4} \sin ^{2} \theta_{L}+F_{L} \cos ^{2} \theta_{K}+\frac{1-F_{L}}{4} \sin ^{2} \theta_{K} \cos 2 \theta_{L}\right. \\
& -F_{L} \cos ^{2} \theta_{K} \cos 2 \theta_{L}+S_{3} \sin ^{2} \theta_{K} \sin ^{2} \theta_{L} \cos 2 \phi \\
& +S_{4} \sin 2 \theta_{K} \sin 2 \theta_{L} \cos \phi+S_{5} \sin 2 \theta_{K} \sin \theta_{L} \cos \phi \\
& +S_{6} \sin ^{2} \theta_{K} \cos \theta_{L}+S_{7} \sin 2 \theta_{K} \sin \theta_{L} \sin \phi \\
& \left.+S_{8} \sin 2 \theta_{K} \sin 2 \theta_{L} \sin \phi+S_{9} \sin ^{2} \theta_{K} \sin ^{2} \theta_{L} \sin 2 \phi\right]
\end{aligned}
$$

where $F_{L}$ is the fraction of longitudinally polarised $K^{* 0}$ and $S_{i}$ are the angular coefficients related to the decay transversity amplitudes. Extracting the full set of these parameters from data fit using the above equation is not possible due to limited statistics. Trigonometric transformations are used to simplify the fit so that terms in Eq. (2.1) drop out. The folding schema used in the analysis follows Ref. [7] and results in four sets of transformations, such that three parameters can be extracted from each of four fits: $F_{L}, S_{3}$ and one of the other $S_{i}$ parameters. As a consequence, $S_{6}$ and $S_{9}$ cannot be extracted from the data.

The $S_{i}$ parameters are sensitive to hadronic form factors, and to reduce the corresponding theoretical uncertainties, the following optimized set of parameters $P_{j}^{(\prime)}$ is used in the analysis:

$$
P_{1}=\frac{2 S_{3}}{1-F_{L}}, \quad P_{2}=\frac{1}{2} \frac{S_{6}}{1-F_{L}}, \quad P_{3}=-\frac{S_{9}}{1-F_{L}}, \quad P_{j=4,5,6,8}^{\prime}=\frac{S_{i=4,5,7,8}}{\sqrt{F_{L}\left(1-F_{L}\right)}} .
$$

Extended unbinned maximum likelihood fits of the distributions of $B^{0}$ candidate mass and angular variables $\cos \theta_{K}, \cos \theta_{L}$, and $\phi$ are performed on the data to extract the angular parameters. A sequential fitting procedure is used. At the first step, only the mass distribution is fitted to extract nuisance parameters: signal and background yields and background mass shapes (signal mass parameters are extracted using the $q^{2}$ ranges of charmonia resonances and fixed in these fits). Then, as second step, the fits are performed to the angular distributions and the parameters of interest $F_{L}$ and $P_{j}^{(\prime)}$ are extracted.

The fit model and procedure have been extensively validated using simulated pseudo-experiments. The fits are done separately in three regions of $q^{2}:[0.04,2.0]$, [2.0, 4.0], and $[4.0,6.0] \mathrm{GeV}^{2}$, as well as in wider overlapping bins $[0.04,4.0],[1.1,6.0],[0.04,6.0] \mathrm{GeV}^{2}$, to facilitate comparison with the results of other experiments and theoretical predictions.

A number of sources of systematic uncertainty affecting the analysis are studied. The methods for determining these uncertainties are based on either a comparison of nominal and modified fit 
results, or on observed fit biases in modified pseudo-experiments. Examples of the most significant sources are: various background contributions producing peaks in the distributions of the angular variables; uncertainty of background angular shapes modelling; detector angular acceptance description; detector alignment and magnetic field calibration uncertainties; effect of intrinsic bias of the maximum likelihood estimator. Overall, the statistical uncertainty is dominant over the systematics.

The distributions of $F_{L}$ and $P_{i}^{(\prime)}$ parameters as functions of $q^{2}$ are shown in Fig. 2. The figure shows predictions of various theoretical approaches based on the SM: Ciuchini et al. (CFFMPSV) [8], Descotes-Genon et al. (DHMV) [9], and Jäger and Martin Camalich (JC) [10]. Results of measurements performed by LHCb [3], CMS [11], Belle [12, 13], and BaBar [14] experiments are also shown.

Theoretical predictions are in a good agreement with the ATLAS result, except for the $P_{4}^{\prime}$ and $P_{5}^{\prime}$ measurements in $q^{2} \in[4.0,6.0] \mathrm{GeV}^{2}$ and $P_{8}^{\prime}$ in $q^{2} \in[2.0,4.0] \mathrm{GeV}^{2}$. The observed deviation of $P_{4}^{\prime}$ and $P_{5}^{\prime}$ from the SM expectations is consistent with that reported by $\mathrm{LHCb}$ [3] and for both parameters amounts to approximately 2.7 standard deviations from the DHMV calculation (with less significant difference for the other predictions). All measurements are found to be within three standard deviations of the range covered by the different predictions.

\section{Measurement of relative width difference of the $B^{0}-\bar{B}^{0}$ system}

The $B^{0}-\bar{B}^{0}$ decay width measurement analysis uses the combined $p p$ collisions dataset collected by ATLAS at $\sqrt{s}=7$ and $8 \mathrm{TeV}$ in 2011-2012 corresponding to integrated luminosities of 4.9 and $20.3 \mathrm{fb}^{-1}$, respectively. The main idea of the measurement is extracting the $\Delta \Gamma_{d}$ from the lifetime-dependent ratio of $B^{0}$ decay rates to $J / \psi K^{* 0}\left(K^{+} \pi^{-}\right)$and $J / \psi K_{S}^{0}\left(\pi^{+} \pi^{-}\right)$final states.

Time-dependent decay rate of $B^{0}$ meson to a final state $f$ can be expressed as

$$
\begin{aligned}
\Gamma[t, f] & \equiv \sigma\left(B^{0}\right) \Gamma\left(B^{0}(t) \rightarrow f\right)+\sigma\left(\bar{B}^{0}\right) \Gamma\left(\bar{B}^{0}(t) \rightarrow f\right) \\
& \propto e^{-\Gamma_{d} t}\left[\cosh \frac{\Delta \Gamma_{d} t}{2}+A_{\mathrm{P}} A_{\mathrm{CP}}^{\mathrm{dir}} \cos \Delta m_{d} t+A_{\Delta \Gamma} \sinh \frac{\Delta \Gamma_{d} t}{2}+A_{\mathrm{P}} A_{\mathrm{CP}}^{\operatorname{mix}} \sin \Delta m_{d} t\right] .
\end{aligned}
$$

Here $t$ is a proper decay time of the $B^{0}$ meson, $\Delta \Gamma_{d}$ and $\Delta m_{d}$ are the difference of widths and masses of two physics mass eigenstates. The $A_{\mathrm{P}}$ is a $B / \bar{B}$ production asymmetry defined as $A_{\mathrm{P}}=$ $\left(\sigma\left(B^{0}\right)-\sigma\left(\bar{B}^{0}\right)\right) /\left(\sigma\left(B^{0}\right)+\sigma\left(\bar{B}^{0}\right)\right)$; other coefficients $A_{\mathrm{CP}}^{\mathrm{dir}}, A_{\mathrm{CP}}^{\mathrm{mix}}, A_{\Delta \Gamma}$ depend on the final state $f$. They are well defined for either flavour-specific or CP eigenstates. Thus, for $J / \psi K^{* 0}$ which is a flavour-specific state,

$$
A_{\mathrm{CP}}^{\mathrm{dir}}=1(-1 \text { for the charge conjugate state }), \quad A_{\mathrm{CP}}^{\mathrm{mix}}=0, \quad A_{\Delta \Gamma}=0,
$$

and for a CP eigenstate $J / \psi K_{S}^{0}$,

$$
A_{\mathrm{CP}}^{\mathrm{dir}}=0, \quad A_{\mathrm{CP}}^{\mathrm{mix}}=-\sin 2 \beta, \quad A_{\Delta \Gamma}=\cos 2 \beta,
$$

where $\beta$ is the angle of the CKM unitarity triangle.

Substituting Eqs. (3.2) and (3.3) into Eq. (3.1), one has for $J / \psi K_{S}^{0}$

$$
\Gamma\left[t, J / \psi K_{S}^{0}\right] \propto e^{-\Gamma_{d} t}\left[\cosh \frac{\Delta \Gamma_{d} t}{2}+\cos 2 \beta \sinh \frac{\Delta \Gamma_{d} t}{2}-A_{\mathrm{P}} \sin 2 \beta \sin \Delta m_{d} t\right],
$$



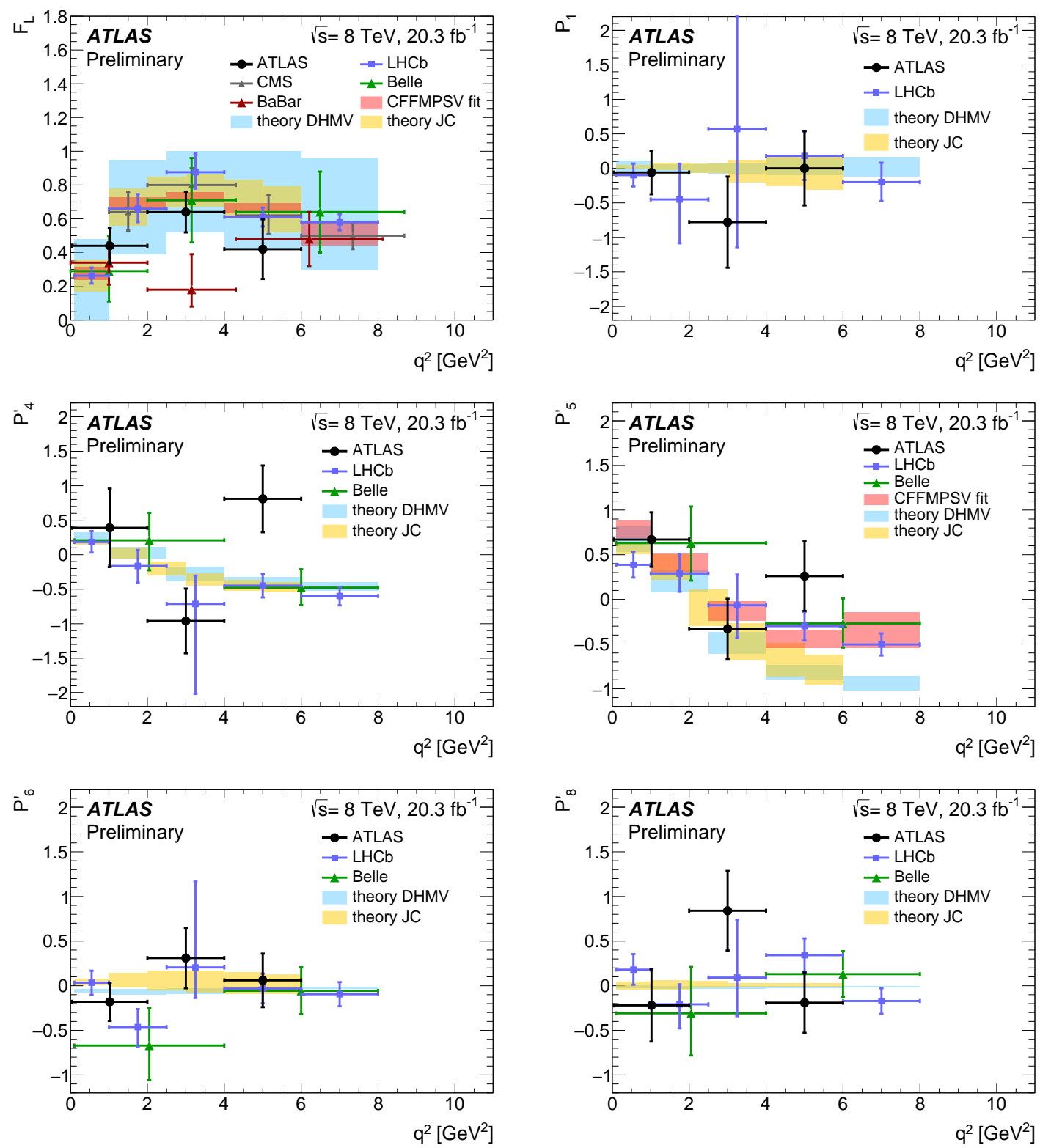

Figure 2: The measured values of $F_{L}$ and $P_{i}^{(\prime)}$ compared with predictions from the theoretical groups and other experiment results. Figure taken from Ref. [2].

and for a sum of $J / \psi K^{* 0}$ and $J / \psi \bar{K}^{* 0}$

$$
\Gamma\left[t, J / \psi K^{* 0}+J / \psi \bar{K}^{* 0}\right] \propto e^{-\Gamma_{d} t} \cosh \frac{\Delta \Gamma_{d} t}{2} .
$$

The ratio between Eqs. (3.4) and (3.5) is used to fit the ratio of time-dependent decay rates to corresponding final states and extract the value of $\Delta \Gamma_{d}$. The sensitivity to $\Delta \Gamma_{d}$ comes from Eq. (3.4) while Eq. (3.5) provides the normalization, which helps to reduce the systematics uncertainties.

To build the inputs for the fit, the signal yields in both $B^{0}$ decay modes are extracted in bins of the meson proper decay length $L_{\text {prop }}^{B}$. This is done by fitting the corresponding decay candidates 
mass distributions in each bin of $L_{\text {prop }}^{B}$. The extracted yields are further corrected for detector acceptances and efficiencies.

In this analysis, the production asymmetry $A_{\mathrm{P}}$ is extracted from data. A time-dependent charge asymmetry of flavour-specific decay $B^{0} \rightarrow J / \psi K^{* 0}$ is used for that. The expected charge asymmetry in $i^{\prime}$ th bin of $L_{\text {prop }}^{B}$ can be expressed as

$$
A_{i, \exp }=\left(A_{\mathrm{det}}+A_{i, \mathrm{osc}}\right)(1-2 W) .
$$

Here $W$ is a dilution coefficient accounting for wrongly identified $B^{0}$ decays when $K^{+}$and $\pi^{-}$are swapped. From simulation it is determined to be $W=0.12 \pm 0.02$ where the uncertainty takes into account possible variations of $B^{0}$ production and decay modelling. Detector-related asymmetry, $A_{\text {det }}$, is caused by differences in reconstruction of positive and negative particles (mainly the kaons). The oscillating component of the asymmetry, $A_{i, \text { osc }}$, is defined by the decay rates of $B^{0}$ to the two charge conjugate states:

$$
\Gamma\left[t, J / \psi K^{* 0}\left(J / \psi \bar{K}^{* 0}\right)\right] \propto e^{-\Gamma_{d} t}\left[\cosh \frac{\Delta \Gamma_{d} t}{2}+(-) A_{\mathrm{P}} \cos \Delta m_{d} t\right] .
$$

Both $A_{\mathrm{det}}$ and $A_{\mathrm{P}}$ are extracted by a $\chi^{2}$ fit of the observed charge asymmetry $A_{\mathrm{obs}}$ distribution shown in Fig. 3 with the expected one (3.6). The observed asymmetry in $i^{\prime}$ th bin of $L_{\text {prop }}^{B}$ is defined as

$$
A_{i, \mathrm{obs}}=\frac{N_{i}\left(J / \psi K^{* 0}\right)-N_{i}\left(J / \psi \bar{K}^{* 0}\right)}{N_{i}\left(J / \psi K^{* 0}\right)+N_{i}\left(J / \psi \bar{K}^{* 0}\right)} .
$$

The fit results into the values

$$
\begin{aligned}
A_{\text {det }} & =(1.33 \pm 0.24 \pm 0.30) \times 10^{-2}, \\
A_{\mathrm{P}} & =(0.25 \pm 0.48 \pm 0.05) \times 10^{-2},
\end{aligned}
$$

where the first uncertainty is statistical and the second is systematics. The measured value of $A_{\mathrm{det}}$ is consistent with results from the simulation of interactions in the detector. The measurement of $B^{0}$ production asymmetry for the kinematic range $p_{\mathrm{T}}\left(B^{0}\right)>10 \mathrm{GeV},\left|\eta\left(B^{0}\right)\right|<2.5$ is consistent with zero, as well as with the LHCb result $A_{\mathrm{P}}=(-0.36 \pm 0.76 \pm 0.28) \times 10^{-2}$ [15] obtained for the kinematic range $4<p_{\mathrm{T}}\left(B^{0}\right)<30 \mathrm{GeV}, 2.5<\eta\left(B^{0}\right)<4.0$.

For the measurement of $\Delta \Gamma_{d}$ with a fit to the ratio between Eqs. (3.4) and (3.5), the $A_{\mathrm{P}}$ value obtained above is used while for $1 / \Gamma_{d}, \Delta m_{d}$, and $\sin 2 \beta$ the world average values [6] are taken. Thus the relative decay width difference $\Delta \Gamma_{d} / \Gamma_{d}$ is left the only free parameter of the fit. Figure 4 shows the efficiency-corrected ratios of $B^{0} \rightarrow J / \psi K_{S}^{0}$ and $B^{0} \rightarrow J / \psi K^{* 0}$ yields as functions of the proper decay length.

The fit results are consistent between $\sqrt{s}=7$ and $8 \mathrm{TeV}$ datasets. The combined result for the whole Run-1 dataset after accounting for all correlations of systematics is

$$
\left.\Delta \Gamma_{d} / \Gamma_{d}=(-0.1 \pm 1.1 \text { (stat.) } \pm 0.9 \text { (syst. })\right) \times 10^{-2} .
$$

Dominant systematics contribution comes from extracting the signal yields in the bins of proper decay length. This corresponds to the uncertainties of the models used for fitting the mass distributions of both decay channel candidates in each $L_{\text {prop }}^{B}$ bin. 


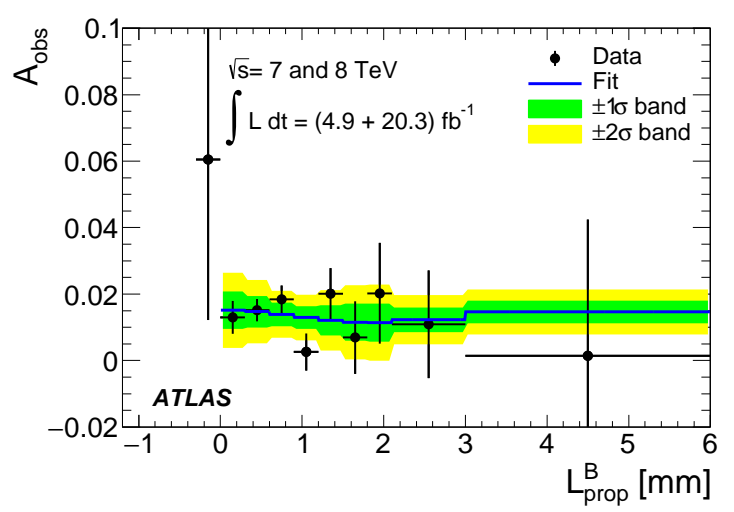

Figure 3: Observed charge asymmetry $A_{\text {obs }}$ in $B^{0} \rightarrow J / \psi K^{* 0}$ decays as a function of the $B^{0}$ proper decay length $L_{\text {prop. }}^{B}$. The line shows the fit result for the expected asymmetry which is caused by the $B^{0}$ production asymmetry $A_{\mathrm{P}}$ and the detector asymmetry $A_{\mathrm{det}}$. The former manifests itself in oscillation across $L_{\mathrm{prop}}^{B}$ while the latter results in a constant offset from zero. The error bands correspond to the combination of uncertainties obtained by the fit for $A_{\mathrm{P}}$ and $A_{\text {det }}$. Figure taken from Ref. [4].
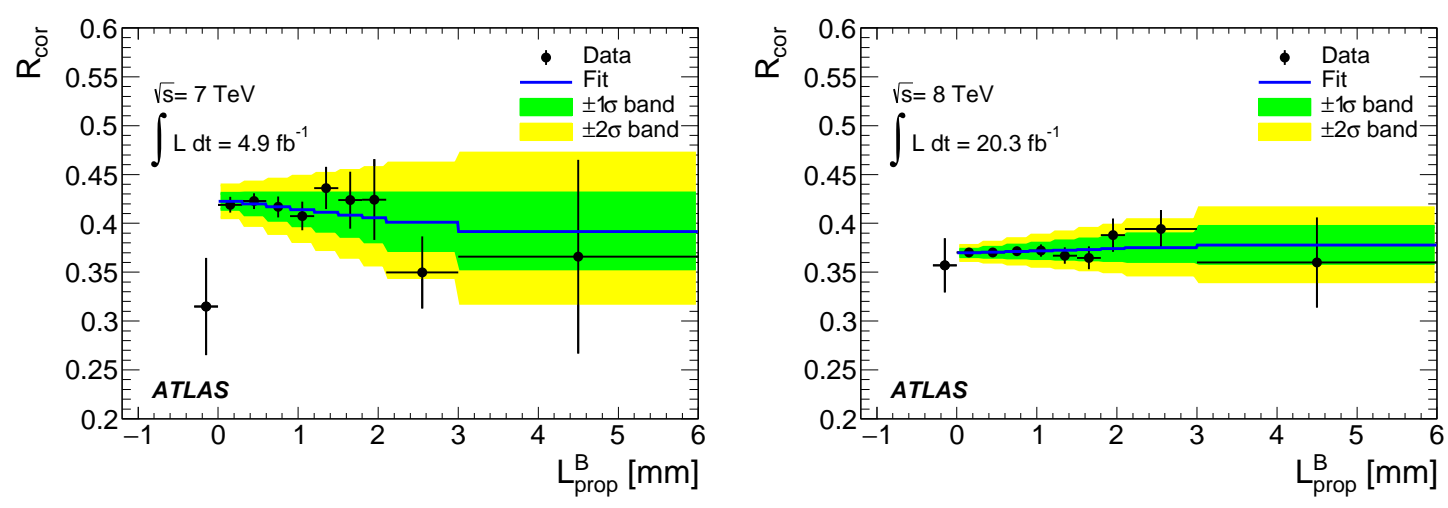

Figure 4: Efficiency-corrected ratio of the observed decay length distributions, $R_{\text {cor }}\left(L_{\text {prop }}^{B}\right)$ for (left) $\sqrt{s}=7 \mathrm{TeV}$ and (left) $\sqrt{s}=8 \mathrm{TeV}$ datasets. The line shows the fit result. The error bands correspond to uncertainties of $\Delta \Gamma_{d} / \Gamma_{d}$ determined by the fit. Figure taken from Ref. [4].

This is the most precise single measurement of this quantity to date. It agrees with the SM prediction, although the measurement uncertainty is still larger by an order of magnitude than the theoretical one. The result is consistent with earlier measurements by BaBar [16], Belle [17], and $\mathrm{LHCb}[18]$.

\section{Summary}

Two heavy flavour decay analyses sensitive to new physics contributions performed by the ATLAS experiment at LHC are presented. No significant deviations from the Standard Model expectations are found. Both measurements use Run-1 data: a sample of $20.3 \mathrm{fb}^{-1}$ collected at $\sqrt{s}=8 \mathrm{TeV}$ in the $B^{0} \rightarrow \mu^{+} \mu^{-} K^{* 0}$ analysis and the full sample of 4.9 and $20.3 \mathrm{fb}^{-1}$ collected at $\sqrt{s}=7$ and $8 \mathrm{TeV}$, respectively, in the $B^{0}-\bar{B}^{0}$ width difference measurement. In both cases the 
uncertainties are dominated by statistical uncertainty. This hints at possible major improvements when these types of measurements are performed with the much larger Run-2 dataset.

\section{References}

[1] ATLAS Collaboration, The ATLAS Experiment at the CERN Large Hadron Collider, JINST 3 (2008) S08003.

[2] ATLAS Collaboration, Angular analysis of $B_{d}^{0} \rightarrow K^{*} \mu^{+} \mu^{-}$decays in pp collisions at $\sqrt{s}=8 \mathrm{TeV}$ with the ATLAS detector, submitted to JHEP, arXiv:1805.04000 [hep-ex] .

[3] LHCb Collaboration, Angular analysis of the $B^{0} \rightarrow K^{* 0} \mu^{+} \mu^{-}$decay using $3 \mathrm{fb}^{-1}$ of integrated luminosity, JHEP 02 (2016) 104, arXiv: 1512.04442 [hep-ex] .

[4] ATLAS Collaboration, Measurement of the relative width difference of the $B^{0}-\bar{B}^{0}$ system with the ATLAS detector, JHEP 06 (2016) 081, arXiv: 1605.07485 [hep-ex ] .

[5] A. Lenz and U. Nierste, Numerical Updates of Lifetimes and Mixing Parameters of B Mesons, in CKM unitarity triangle. Proceedings, 6th International Workshop, CKM 2010, Warwick, UK, September 6-10, 2010. 2011. arXiv:1102.4274 [hep-ph].

[6] K. A. Olive et al. (Particle Data Group), Review of Particle Physics, Chin. Phys. C 38 (2014) 090001.

[7] LHCb Collaboration, Measurement of Form-Factor-Independent Observables in the Decay $B^{0} \rightarrow K^{* 0} \mu^{+} \mu^{-}$, Phys. Rev. Lett. 111 (2013) 191801, arXiv:1308.1707 [hep-ex] .

[8] M. Ciuchini, M. Fedele, E. Franco, S. Mishima, A. Paul, L. Silvestrini, and M. Valli, $B \rightarrow K^{*} \ell^{+} \ell^{-}$ decays at large recoil in the Standard Model: a theoretical reappraisal, JHEP 06 (2016) 116, arXiv:1512.07157 [hep-ph].

[9] S. Descotes-Genon, L. Hofer, J. Matias, and J. Virto, On the impact of power corrections in the prediction of $B \rightarrow K^{*} \mu^{+} \mu^{-}$observables, JHEP 12 (2014) 125, arXiv:1407.8526 [hep-ph] .

[10] S. Jäger and J. Martin Camalich, On B $\rightarrow V \ell \ell$ at small dilepton invariant mass, power corrections, and new physics, JHEP 05 (2013) 043, arXiv: 1212.2263 [hep-ph] .

[11] CMS Collaboration, Angular analysis of the decay $B^{0} \rightarrow K^{* 0} \mu^{+} \mu^{-}$from pp collisions at $\sqrt{s}=8 \mathrm{TeV}$, Phys. Lett. B 753 (2016) 424-448, arXiv: 1507.08126 [hep-ex] .

[12] J. T. Wei et al., Measurement of the Differential Branching Fraction and Forward-Backword Asymmetry for $B \rightarrow K^{(*)} \ell^{+} \ell^{-}$, Phys. Rev. Lett. 103 (2009) 171801, arXiv: 0904.0770 [hep-ex].

[13] S. Wehle et al., Lepton-Flavor-Dependent Angular Analysis of $B \rightarrow K^{*} \ell^{+} \ell^{-}$, Phys. Rev. Lett. 118 (2017) 111801, arXiv:1612.05014 [hep-ex].

[14] J. P. Lees et al., Measurement of angular asymmetries in the decays $B \rightarrow K^{*} \ell^{+} \ell^{-}$, Phys. Rev. D 93 (2016) 052015, arXiv:1508.07960 [hep-ex] .

[15] LHCb Collaboration, Measurement of the $\bar{B}^{0}-B^{0}$ and $\bar{B}_{s}^{0}-B_{s}^{0}$ production asymmetries in $p p$ collisions at $\sqrt{s}=7 \mathrm{TeV}$, Phys. Lett. B 739 (2014) 218-228, arXiv:1408.0275 [hep-ex].

[16] B. Aubert et al., Limits on the decay rate difference of neutral-B mesons and on CP, T, and CPT violation in $B^{0} \bar{B}^{0}$ oscillations, Phys. Rev. D 70 (2004) 012007, arXiv: hep-ex/ 0403002.

[17] T. Higuchi et al., Search for Time-Dependent CPT Violation in Hadronic and Semileptonic B Decays, Phys. Rev. D 85 (2012) 071105, arXiv:1203.0930 [hep-ex] .

[18] LHCb Collaboration, Measurements of the $B^{+}, B^{0}, B_{s}^{0}$ meson and $\Lambda_{b}^{0}$ baryon lifetimes, JHEP 04 (2014) 114, arXiv:1402.2554 [hep-ex] . 